\title{
Evaluación del uso de equipo de protección personal (EPP) en personal de salud de un Hospital Universitario mediante las curvas CUSUM
}

\author{
Evaluation of the use of personal protective equipment (PPE) in health \\ personnel of a University Hospital using the CUSUM curves
}

José Ricardo Navarro-Vargas ${ }^{1, *}$, Gloria Yasmín Mesías-Castillo², Itali Linero-Segrera³ , Diana Marcela Bejarano-Villamarín , Gina Elizabeth Ramírez-Bedoya ${ }^{5}$, Ingrid Jany-Guzmán ${ }^{5}$, Análida Elizabeth Pinilla-Roa ${ }^{6}$

Decanatura Facultad de Medicina, Universidad Nacional de Colombia.

Directora Unisalud Bogotá, Colombia.

Facultad de Odontología, Universidad Nacional de Colombia. Bogotá, Colombia.

Facultad de Medicina, Universidad Nacional de Colombia. Bogotá, Colombia.

Oficina de Calidad, Hospital Universitario Nacional de Colombia. Bogotá, Colombia.

6 Hospital Universitario Nacional. Bogotá, Colombia.

Facultad de Medicina, Universidad Nacional de Colombia. Subdirección de Educación, Hospital Universitario Nacional. Bogotá, Colombia.

Los autores no refieren conflicto de interés.

Los autores no refieren financiación.

Fecha de recepción: 25 de octubre de 2021 / Fecha de aceptación: 12 de noviembre de 2021

\begin{abstract}
Introduction: Health personnel who care for patients with COVID-19 are at great risk of contagion, unless they learn and properly implement the procedure for donning and doffing personal protective equipment (PPE). Objectives: To evaluate the use of PPE (donning and doffing) using the CUSUM method in health personnel who care for hospitalized patients with a presumptive or confirmed diagnosis of COVID-19. Use the CUSUM curves as an appropriate method for the meaningful learning and psychomotor skills of these professionals. Materials and Methods: Cross-sectional study carried out in 10 health professionals from the National University Hospital. Measurements were made for one hour per participant from July 13 to 29, 2020, and in each one checklists were used to evaluate compliance with the protocol. In the formative evaluation using the CUSUM, an acceptable failure rate of $20 \%$ (Po) was considered and an unacceptable one of $40 \%$ (P1), with an error parameter $\alpha$ and $\beta$ of $10 \%$. Results: $40 \%$ reached $\mathrm{HO}$, the goal; $20 \%$ approached the target and $40 \%$ and $20 \%$ were distanced from the target with regard to donning and doffing the PPE. Conclusions: Proper use of PPE is a strategy for the prevention of contagion of COVID-19 in health personnel; The study found that less than half demonstrated adequate use, so training these professionals in the placement and removal of PPE according to an institutional protocol based on constant feedback and strict monitoring is essential to achieve the acquisition of skills, which will considerably reduce your risk of infection in clinical practice.
\end{abstract}

Key words: Learning curve, checklist, formative feedback, critical incident stress debriefing, evaluation, personal protective equipment.

\section{RESUMEN}

Introducción: El personal de salud que atiende a pacientes con COVID-19 está en un gran riesgo de contagio, a menos que aprenda e implemente apropiadamente el procedimiento de colocación y retiro de los elementos de protección personal (EPP). Objetivos: Evaluar el uso de EPP (colocación y retiro) mediante el método CUSUM en personal de la salud que presta atención 
a pacientes hospitalizados con diagnóstico presuntivo o confirmado de COVID-19. Utilizar las curvas CUSUM como método apropiado para el aprendizaje significativo y las habilidades psicomotoras de estos profesionales. Materiales y Métodos: Estudio transversal realizado en 10 profesionales de la salud del Hospital Universitario Nacional. Las mediciones se realizaron por dos horas por participante del 13 al 29 de julio de 2020, y en cada una se utilizaron listas de chequeo para la evaluación del cumplimiento del protocolo. En la evaluación formativa mediante las CUSUM, se consideró un porcentaje de fallo aceptable del $20 \%$ (PO) y uno inaceptable del $40 \%$ (P1), con un parámetro de error $\alpha$ y $\beta$ del 10\%. Resultados: $40 \%$ alcanzó el $H_{0}$ el objetivo; $20 \%$ se acercó al objetivo y $40 \%$ y $20 \%$ estuvieron distanciados del objetivo con respecto a la colocación y retiro del EPP. Conclusiones: El uso apropiado de EPP es una estrategia de prevención de contagio de COVID-19 en personal de la salud; el estudio encontró que menos de la mitad demostraron un uso adecuado, por lo que la capacitación de estos profesionales en la colocación y retiro de PPE según un protocolo institucional basado en la retroalimentación constante y un seguimiento estricto, es fundamental para lograr la adquisición de las habilidades, lo que reducirá considerablemente su riesgo de contagio en la práctica clínica.

Palabras clave: Curva de aprendizaje, lista de verificación, retroalimentación formativa, debriefing, evaluación, equipo de protección personal (DeCS).

\section{Introducción}

A finales de 2019 se identificó que el nuevo coronavirus SARS-CoV-2 era el responsable de una serie de casos de neumonía atípica en Wuhan, China. La infección por este virus pasó rápidamente de ser un brote aislado en una región de China a convertirse en una emergencia de salud pública mundial[1); el 11 de marzo de 2020 la OMS declaró la enfermedad producida por este virus, Coronarivirus disease 2019 (COVID-19) como pandemia[2],[3]. Al 29 de julio de 2020, Colombia registraba 257.101 casos y 8.777 defunciones, ocupando el puesto 15 en el mundo y el 6 en la región[4]; en esta misma fecha, el Instituto Nacional de Salud[5] confirmó 4.116 profesionales de la salud contagiados, de los cuales 34 habían fallecido, 3.443 se habían recuperado y 639 permanecían en aislamiento. Los más afectados eran 1.398 auxiliares de enfermería, 685 médicos, 566 enfermeros, 358 administrativos y 181 empleados de aseo y alimentación. El último reporte del Ministerio de Salud (2 de julio de 2021) confirma 4.297.302 casos positivos y 107.723 fallecidos por SARS-CoV-2, mientras a nivel mundial estas cifras son de 182.875 .305 y 3.959 .251 respectivamente[6].

EN cuanto a su transmisión, el virus SARS-CoV-2 entra por las membranas mucosas, mediante gotículas y aerosoles que pasan por contacto persona a persona; además, puede sobrevivir en varias superficies durante horas o días[7)].

Los trabajadores de la salud, por ser la primera línea de respuesta, son la población que está en mayor riesgo de contraer y transmitir la enfermedad[8], lo que hace imperativo el cumplimiento de los protocolos de bioseguridad para colocación, uso, retiro y desecho de los elementos de protección personal (EPP) [9]. Adicionalmente, las instituciones prestadoras de salud (IPS) deben garantizar la disponibilidad de recursos para la prevención de infecciones, tomar las medidas de control necesarias para proporcionar la seguridad de los trabajadores de la salud y proveer la capacitación sobre la técnica de colocación y retiro de los EPP[10].

De acuerdo con el Center for Disease Control and Prevention (CDC) y la Occupational Safety and Health Administration (OSHA), los EPP son la ropa o conjunto de elementos especiales para la protección contra agentes infecciosos, por lo cual, el personal de salud los deberá utilizar correctamente, según el grado de riesgo[8], durante la atención de pacientes en el mar- co de esta pandemia, debido a la alta tasa de transmisibilidad del virus SARS-CoV-2.

Desde antes de la pandemia se han publicado investigaciones orientadas a mejorar la utilización de los EPP, las cuales resaltan que su uso óptimo es difícil e inadecuado[11],[12], aunque es una de las estrategias más importantes para proteger de la contaminación y evitar la propagación de agentes patógenos a pacientes[11]-[13]. En la mayoría de los casos deriva en autocontaminación por el uso deficiente[11],[14], en especial durante el retiro[14]. Además, se evidencia que los profesionales de salud requieren de un conocimiento unificado al respecto y de suficiente capacitación técnica para la colocación, retiro y uso de los EPP[12],[15].

En el mundo, las instituciones de salud enfocan sus esfuerzos en optimizar el uso de EPP mediante estrategias que generen mayor adherencia a los protocolos y mejores prácticas[11]. Se ha demostrado que estandarizar la capacitación para la colocación y el retiro de los EPP mediante simulación e instrucciones cara a cara, pueden mejorar significativamente los indicadores de desempeño para evaluar esta competencia; con estas acciones se disminuye la ocurrencia de errores críticos y no críticos y se minimiza la posibilidad de contaminación[12],[13].

El CUSUM es un tipo de gráfico de control, que muestra los cambios en el desempeño del procedimiento o del proceso de aprendizaje a través del tiempo.

En el área de la salud, las curvas CUSUM se consideran un método estadístico que sirve para predecir una probabilidad de éxito en términos de mínima falla (mínimo error) y alta tasa de éxitos en la realización de procedimientos o habilidades psicomotoras en el desempeño clínico. Igualmente, permiten vigilar la evolución del aprendizaje y determinar el punto en el cual, según probabilidades, el participante logra realizar los procedimientos con mayor seguridad, lo cual implica un menor riesgo para él y para los pacientes[16]. Toman en cuenta las posibilidades de falsos positivos o error tipo 1, o los falsos negativos o error tipo 2, que se deben a la subjetividad del evaluador.

De acuerdo con Starkie y Drake[17], las curvas CUSUM son una gran herramienta de control de calidad para profesionales capacitados, como en este caso, donde todos los participantes han recibido una capacitación previa. Tiene como ventajas la sencillez en su aplicación, la fácil interpretación visual y la capacidad para detectar cambios en las tendencias; sin embargo, no se logra identificar la causa de la desviación que se pueda 
presentar durante el aprendizaje, de ahí que se requiera hacer un seguimiento estrecho a cada participante para un verdadero aprendizaje significativo[18].

La confidencialidad y la confiabilidad psicométrica del estudio dependen de que haya varios evaluadores que aplican las curvas CUSUM de forma independiente[19]. Los factores sicológicos pueden influir en los participantes, y al tener varias evaluaciones por distintos evaluadores se garantiza una independencia estadística[20]. Se ha descrito alta variación interindividual en la adquisición de una habilidad psicomotora en prácticas de anestesia; factores como la institución donde se realiza la formación y el número de casos a los cuales se expone el participante, pueden ser decisivos en el aprendizaje[21].

Teniendo en cuenta lo anterior, el objetivo del presente estudio fue evaluar el uso de EPP (colocación y retiro) mediante el método de curvas de aprendizaje de sumatoria acumulada (CUSUM) en personal de la salud que presta atención a pacientes hospitalizados con diagnóstico sospechado o confirmado de COVID-19 en un hospital universitario de Bogotá D.C., Colombia.

\section{Materiales y Métodos}

Estudio transversal (tipo serie de casos) realizado del 13 al 29 de julio de 2020 en 10 profesionales de la salud que se encontraban laborando en el área COVID-19 del servicio de Hospitalización del Hospital Universitario Nacional de Colombia, distribuidos de la siguiente manera:

- 2 profesionales de enfermería.

- 3 profesionales de fisioterapia.

- 3 médicos hospitalarios.

- 2 auxiliares de enfermería.

Previo a la evaluación, todos los participantes recibieron la misma capacitación en una sesión educativa presencial y en un video institucional, y aceptaron participar de este simulacro formativo de forma voluntaria. Las mediciones se realizaron en dos (2) horas por participante durante 3 semanas.

El desempeño de cada participante tanto en la secuencia de colocación, como en la secuencia de retiro del EPP se midió 8 veces, para un total de 160 mediciones; las evaluaciones fueron realizadas por las instructoras de los pasos de colocación y retiro mediante observación directa, siguiendo el protocolo de contingencia institucional (TH-PT-07 que reposa en el aplicativo DARUMA del HUN). En cada medición, se utilizaron listas de chequeo (TH-FR-85) (ver Anexo 1 y 2 en https://revistachilenadeanestesia.cl/PII/anexo-revchilanestv5128121113.pdf) para evaluar el conocimiento sobre del paciente, lavado de manos, secuencia de los pasos y adecuada colocación y retiro de EPP.

Se ha reportado que las curvas porcentuales y las CUSUM, las cuales facilitan la evaluación formativa, son útiles para evaluar habilidades psicomotoras en personal de la salud (22), puesto que después que el profesional de la salud adquiere el conocimiento declarativo y procedimental (niveles 1 y 2 de la Pirámide de Miller), las CUSUM permiten evaluar de forma personalizada el "saber cómo" mediante la puesta en práctica de dicho conocimiento en un laboratorio de simulación (nivel 3), para que de esta forma adquiera la competencia adecuadamente y la pueda poner en práctica a la hora de prestar atención médica al paciente (nivel 4)[16],[23]. Teniendo en cuenta lo anterior, las CUSUM fueron utilizadas como estrategia de evaluación formativa en este estudio.

Los protocolos de uso de EPP a evaluar fueron dos: secuencia de colocación y de retiro de EPP. La colocación consta de 13 pasos y el retiro, de 10 pasos.

Secuencia de colocación de EPP:

1. Retirar todas las prendas personales (joyas, reloj, entre otros).

2. Verificar kit de EPP completo (bata, mascarilla, gorro, monogafas, guantes, protector facial).

3. Ingresar a la antecámara o al espacio destinado para este fin.

4. Realizar higiene de manos.

5. Colocar de gorro.

6. Colocar mascarilla.

7. Colocar monogafas.

8. Colocar protector facial.

9. Colocar guantes de manejo (A).

10. Colocar bata antifluidos.

11. Ingresar a la habitación.

12. Colocar guantes de manejo (B).

13. Realizar atención o procedimiento.

Secuencia de retiro de EPP:

1. Retirar guantes de manejo (B).

2. Retirar bata antifluidos.

3. Salir de la habitación.

4. Retirar guantes de manejo (A).

5. Realizar higiene de manos.

6. Colocar guantes de manejo.

7. Retirar el protector facial y desinfectar. Guardar la careta limpia y seca en bolsa rotulada.

8. Retirar monogafas y desinfectar. Guardarlas limpias y secas.

9. Retirar guantes de manejo.

10. Realizar higiene de manos.

Para cada participante se registró, en una base de datos de excel, el cumplimiento de los 13 pasos de colocación y los 10 pasos de retiro de los EPP, según lo reportado en la lista de chequeo para cada medición. El no cumplimiento de un solo paso fue informado como no cumplimiento del protocolo o secuencia.

Para la evaluación formativa de cada persona mediante la construcción de las CUSUM, según los protocolos de colocación y retiro de EPP, se determinó un porcentaje de fallo aceptado (P0) del $20 \%$ y un porcentaje de fallo inaceptable (P1) del $40 \%$; además, se consideró $10 \%(0,1)$ de fallo para los errores estadísticos tipo 1 (Alfa) o falsos positivos y tipo 2 (Beta) o falsos negativos. El registro y análisis de los datos para la obtención de los resultados se realizó mediante un sistema de información que recolectaba los datos y los procesaba de acuerdo con la fórmula CUSUM, a saber:[16]

$s=\mathrm{Q} / \mathrm{P}+\mathrm{Q}$

$P=\operatorname{Ln}(\mathrm{p} 1 / \mathrm{p} 0)$ se calcula con \% de fallo aceptable e inaceptable

$Q=\operatorname{Ln}((1-p 0) /(1-p 1))$

$H_{0}=$ menos $b /(P+Q)$

$b=\operatorname{Ln}((1-a) / b)$

$H_{1}=a /(P+Q)$

$a=\operatorname{Ln}((1-b) / a)$

La curva de aprendizaje se mantiene en la franja entre Ho, 
límite inferior cuando se alcanza el objetivo y H1, límite superior cuando el participante se sale de los parámetros normales para su aprendizaje y deben buscarse causas externas al modelo de aprendizaje que expliquen su desempeño. Por otra parte, el valor p0 (20\%) equivale al porcentaje de fallo aceptable y p1 (40\%) fallo inaceptable.

El estudio contó con la aprobación del Comité de Ética del HUN (ID: CEl-2021-05-02) y siguió los principios éticos de investigación biomédica en seres humanos de la Declaración de Helsinki[24] y las normas técnicas y administrativas de investigación en salud de la resolución 8.430 de 1993 del Ministerio de Salud de Colombia. Además, se contó con consentimiento informado por parte de todos los participantes (Anexo 2).

\section{Resultados}

Respecto a la colocación de EPP, el $40 \%$ de participantes alcanzaron el $\mathrm{H}_{0}$ (No. CUSUM), el $20 \%$ de los participantes se acercaron al objetivo y el otro $40 \%$ estuvieron distanciados del objetivo. En cuanto al retiro del EPP, $40 \%$ de los participantes alcanzaron el objetivo, 20\% estuvieron cerca, 20\% tuvieron un comportamiento regular y $20 \%$ estuvieron lejos del objetivo (Figuras 1 y 2). También se identificó que no hubo una relación directa entre la adquisición de habilidades y la profesión en salud de los participantes.

En el análisis independiente de cada participante, se eviden- ció que hubo personas con 3 o 4 evaluaciones insatisfactorias en la colocación o retiro del EPP, lo que mostró la necesidad de hablar con ellos y definir las acciones para mejorar su competencia en este protocolo (Tablas 1 y 2).

Se debe tener en cuenta que las CUSUM son personalizadas; por lo tanto, no hay un número de repeticiones único para adquirir la habilidad, el aprendizaje depende de las habilidades de cada persona y de sus conocimientos previos.

\section{Discusión}

En el presente estudio, la implementación de la evaluación formativa mediante las CUSUM permitió reconocer el nivel de adquisición de cada participante de las habilidades psicomotoras requeridas para el manejo seguro de EPP[25]. Así mismo, se evidenció que el uso de la lista de chequeo no es suficiente para identificar si las personas han adquirido la habilidad para la colocación y retiro de los EPP.

El personal de salud que atiende personas con infección sospechosas o confirmada por el SARS-CoV-2 está enormemente expuesto al contagio, y es altamente recomendable que implemente de manera rigurosa todas las precauciones para protegerse mediante el uso de EPP[9],[10].

La educación está directamente relacionada con los apropiados comportamientos sanitarios y la disminución de los riesgos de infección[26]. El uso correcto y suficiente de EPP no solo

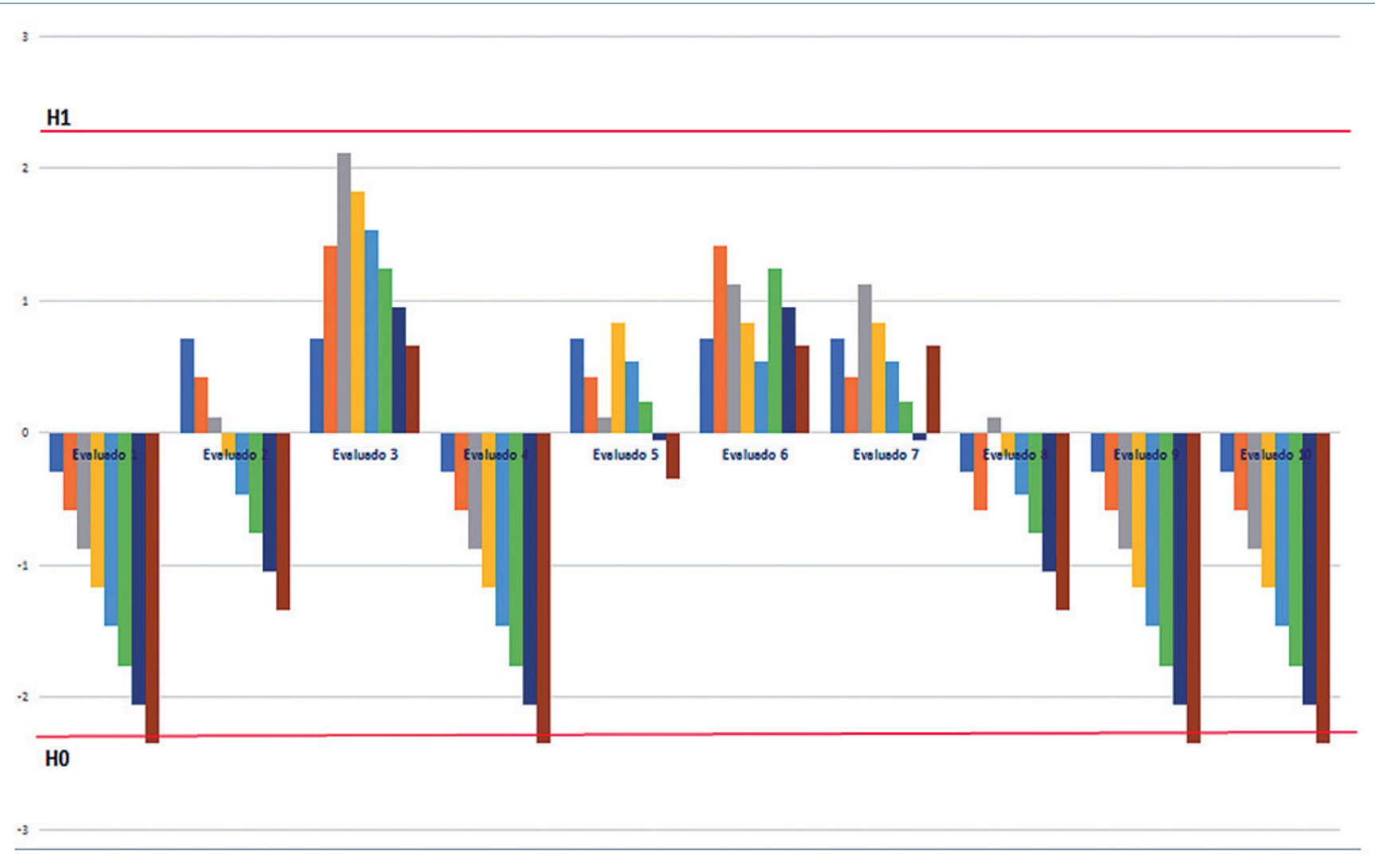

Figura 1. Resultados de aplicación de la lista de chequeo del Protocolo de Colocación de EPP en casos probables o confirmados de COVID-19 (cada barra corresponde a un participante diferente) Fuente: Propia. 
Artículo Original

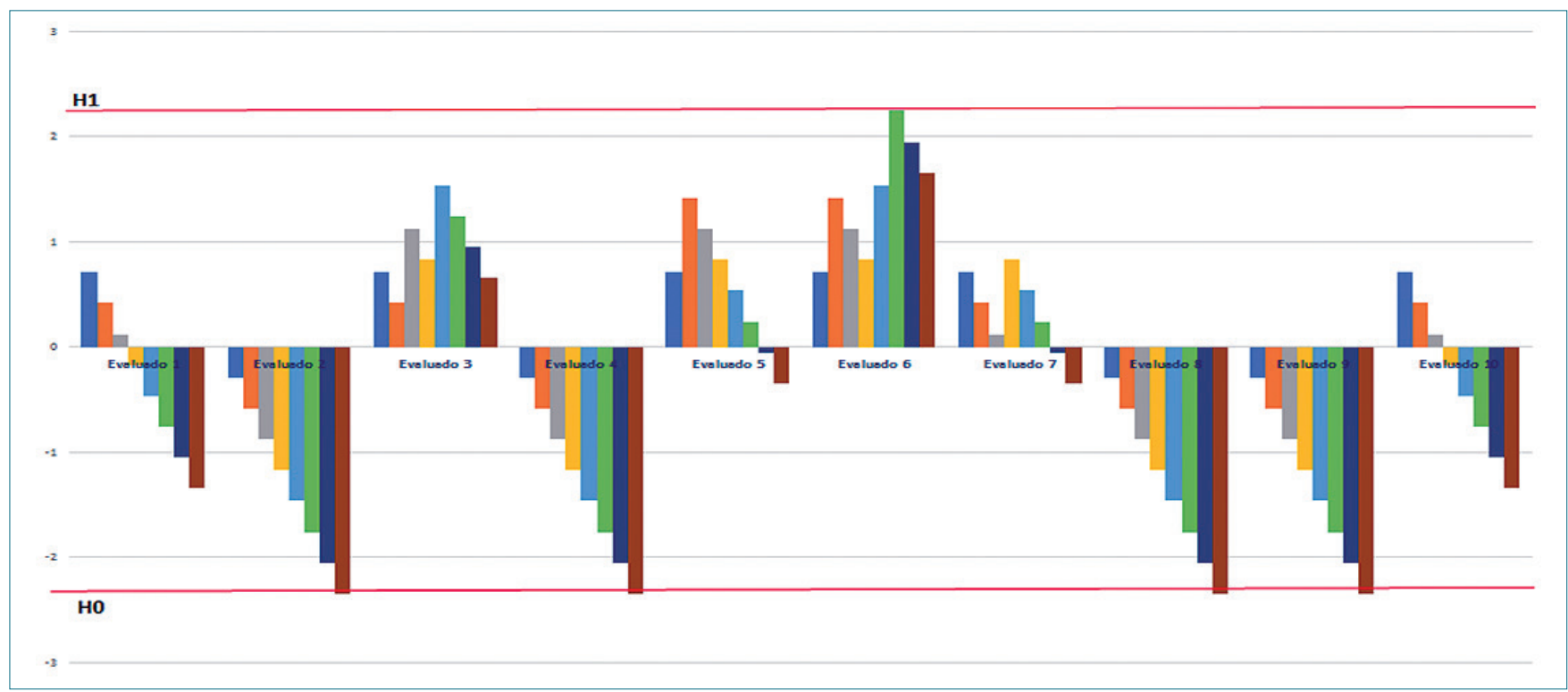

Figura 2. Resultados aplicación de la lista de chequeo del Protocolo de retiro de EPP en casos probables o confirmados de COVID-19 (cada barra corresponde a un participante diferente) Fuente: Propia.

\begin{tabular}{|c|c|c|c|c|c|c|c|c|}
\hline Evaluación & $\# 1$ & \# 2 & \# 3 & $\# 4$ & $\# 5$ & \# 6 & $\# 7$ & $\# 8$ \\
\hline 1 & No cumple & No cumple & Cumple & No cumple & Cumple & Cumple & Cumple & Cumple \\
\hline 2 & Cumple & Cumple & Cumple & Cumple & Cumple & Cumple & Cumple & Cumple \\
\hline 3 & Cumple & Cumple & Cumple & Cumple & Cumple & Cumple & Cumple & Cumple \\
\hline 4 & No cumple & No cumple & Cumple & Cumple & Cumple & No cumple & Cumple & Cumple \\
\hline 5 & No cumple & Cumple & Cumple & Cumple & Cumple & Cumple & Cumple & Cumple \\
\hline 6 & No cumple & No cumple & Cumple & Cumple & Cumple & Cumple & Cumple & Cumple \\
\hline 7 & Cumple & Cumple & No cumple & Cumple & Cumple & Cumple & Cumple & Cumple \\
\hline 8 & No cumple & No cumple & No cumple & Cumple & Cumple & Cumple & Cumple & Cumple \\
\hline 9 & Cumple & Cumple & Cumple & Cumple & Cumple & Cumple & Cumple & Cumple \\
\hline 10 & Cumple & Cumple & Cumple & Cumple & Cumple & Cumple & Cumple & Cumple \\
\hline
\end{tabular}

Fuente: propia

\begin{tabular}{|c|c|c|c|c|c|c|c|c|}
\hline \multirow[t]{2}{*}{ Participante } & Evaluación & & & & & & & \\
\hline & $\# 1$ & $\# 2$ & $\# 3$ & $\# 4$ & \# 5 & \# 6 & $\# 7$ & \# 8 \\
\hline 1 & No cumple & Cumple & Cumple & Cumple & Cumple & Cumple & Cumple & Cumple \\
\hline 2 & Cumple & Cumple & Cumple & Cumple & Cumple & Cumple & Cumple & Cumple \\
\hline 3 & Cumple & Cumple & No cumple & Cumple & No cumple & Cumple & Cumple & Cumple \\
\hline 4 & No cumple & Cumple & Cumple & No cumple & Cumple & Cumple & Cumple & Cumple \\
\hline 5 & No cumple & No cumple & Cumple & Cumple & Cumple & Cumple & Cumple & Cumple \\
\hline 6 & No cumple & No cumple & Cumple & Cumple & No cumple & No cumple & Cumple & Cumple \\
\hline 7 & Cumple & Cumple & No cumple & Cumple & Cumple & Cumple & Cumple & Cumple \\
\hline 8 & No cumple & No cumple & No cumple & Cumple & Cumple & Cumple & Cumple & Cumple \\
\hline 9 & Cumple & Cumple & Cumple & Cumple & Cumple & Cumple & Cumple & Cumple \\
\hline 10 & Cumple & Cumple & Cumple & Cumple & Cumple & Cumple & Cumple & Cumple \\
\hline
\end{tabular}

Fuente: Propia 
contribuye a prevenir la enfermedad por el COVID-19 sino también a reforzar en el equipo de la salud que se está atendiendo al paciente con profesionalismo y se está evitando el contagio a toda persona que entra en contacto con dicho personal de la salud[27].

El aporte principal de este estudio, de acuerdo a la herramienta CUSUM que se usó en la evaluación de los participantes, es que sólo el $40 \%$ de ellos cumplieron con los protocolos, lo cual los expone a un mayor riesgo de contagio por el uso inapropiado de los EPP, y la segunda enseñanza, es que es necesario buscar la causa de la falta de adherencia a los protocolos y reforzar el aprendizaje.

Este trabajo da continuidad al proceso institucional del HUN que busca garantizar la seguridad del personal de salud, profesionales en formación, familias, pacientes y cuidadores, dentro del marco de la emergencia sanitaria que se vive actualmente por la pandemia por COVID-19[7]-[9]. En tal sentido, esta propuesta podrá convertirse en un proyecto interinstitucional de evaluación formativa de diversas facultades y programas académicos de la UNAL y de Unisalud, como el inicio de una trasformación cultural, sustentada en un nuevo enfoque pedagógico que motive a cada participante a cultivar el autocuidado, a cumplir los pasos de los procedimientos de uso de EPP a conciencia, considerando el riesgo real de ser infectado o de infectar a los demás.

\section{Limitaciones}

Una de las limitaciones de este estudio es la cantidad limitada de participantes, sin embargo se contó con un escenario apropiado y con suficiencia de equipos de protección personal y el software de la S.C.A.R.E para la aplicación de las curvas CUSUM. Es posible que en este trabajo de investigación haya potenciales sesgos de información y de selección.

\section{Conclusiones}

El uso de EPP es una excelente estrategia de prevención de contagio de COVID-19 en personal de la salud, lo que lo constituye un factor protector no solo para los trabajadores de la salud, sino para los pacientes y sus familias. Lamentablemente en el presente estudio, poco menos de la mitad, demostraron un uso adecuado, por lo que la capacitación de estos profesionales en la colocación y retiro de PPE según un protocolo institucional basado en la retroalimentación constante y un seguimiento estricto es fundamental para lograr la adquisición de las habilidades necesarias para su uso adecuado, lo que reducirá considerablemente su riesgo de contagio en la práctica clínica.

La evaluación formativa mediante las curvas CUSUM demuestra ser un método apropiado para verificar el aprendizaje significativo y las habilidades psicomotoras del personal en salud que entra en contacto con los pacientes, en el marco de esta pandemia.

Agradecimientos: Un especial reconocimiento al ingeniero de sistemas Édgar Ramírez Novoa, gerente del Departamento de Tecnología de Información de la Sociedad Colombiana de Anestesiología y Reanimación S.C.A.R.E.

\section{Referencias}

1. Mojica-Crespo R, Morales-Crespo MM. [Pandemic COVID-19, the new health emergency of international concern: A review]. Semergen. 2020 Aug;46(1 Suppl 1):65-77. https://doi. org/10.1016/j.semerg.2020.05.010 PMID:32425491

2. Organización Mundial de la Salud. Alocución de apertura del Director General de la OMS en la rueda de prensa sobre la COVID-19 celebrada el 11 de marzo de 2020 [internet]. 11 de marzo 2020 [citado: 12-08-2020]. Disponible en: https://www.who.int/ es/dg/speeches/detail/who-director-general-s-opening-remarksat-the-media-briefing-on-covid-19---11-march-2020

3. Organización Mundial de la Salud. Rolling update on Coronavirus Disease (COVID-19) [internet]. Publicado inicialmente el 31 de diciembre de 2019, actualizable día a día [citado: 2020 jul. 29]. Disponible en: https://www.who.int/emergencies/diseases/novelcoronavirus-2019/events-as-they-happen.2020

4. Organización Panamericana de la Salud. Reporte de Situacion COVID-19 Colombia No. 110-27 de julio de 2020 [internet]. 2020 [citado: 12-08-2020]. Disponible en: https://www.paho. org/es/documentos/reporte-situacion-covid-19-colombia-no104-16-julio-2020

5. Instituto Nacional de Salud (INS). (2020). COVID-19 en personal de salud en Colombia. Boletín No. 28 [internet]. 24 de julio de 2020 [citado: 13-08-2020]. Disponible en: https://www.ins.gov. co/Noticias/paginas/coronavirus.aspx

6. Coronavirus en Colombia. Revisado 2 de julio de 2021 https://colombia.as.com/colombia/2021/07/02/actualidad/1625225782_364983.html

7. Woolleya R, Arumugam S. Personal Protective Equipment (PPE) Guidelines, adaptations and lessons during the COVID-19 pandemic. Ethics, Medicine and Public Health [internet]. 2020;14 [citado: 17-08-2020]. Disponible en: https://doi.org/10.1016/j. jemep.2020.100546

8. Asociación Colombiana de Infectología (ACIN). Consenso Colombiano de atención, diagnóstico y manejo de la infección por SARS-CoV2-COVID-19 en establecimientos de atención de la salud. Recomendaciones basadas en consenso de expertos e informadas en la evidencia. Revista de la Asociación Colombiana de Infectología. 2020;24(3)-S1. https://doi.org/10.22354/ in.v24i3.851.

9. Ministerio de Salud y Protección Social de Colombia. Lineamientos para kit de elementos básicos de protección personal para personal de la salud [internet]. Junio de 2020 [citado: 17-082020]. Disponible en: https://www.minsalud.gov.co/Ministerio/ Institucional/Procesos\%20y\%20procedimientos/GMTG16.pdf

10. Ministerio de Salud y Protección Social de Colombia. Recomendaciones de EPP para personal de salud según el área de atención para COVID-19. Consenso IETS-ACIN [internet]. Febrero 2020 [citado: 18-08-2020]. Disponible en: https://www.minsalud.gov. $\mathrm{co} /$ sites/rid/Lists/BibliotecaDigital/RIDE/NS/tabla-epp-consenso.pdf

11. Reddy SC, Valderrama AL, Kuhar DT. Improving the use of personal protective equipment: applying lessons learned. Clin Infect Dis. 2019 Sep;69 Suppl 3:S165-70. https://doi.org/10.1093/cid/ ciz619 PMID:31517978

12. Casalino E, Astocondor E, Sánchez JC, Díaz-Santana DE, Del Aguila C, Carrillo JP. Personal protective equipment for the Ebola virus disease: A comparison of 2 training programs. Am J Infect Control. 2015 Dec;43(12):1281-7. https://doi.org/10.1016/j. ajic.2015.07.007 PMID:26277572 
13. Verbeek JH, Rajamaki B, ljaz S, Sauni R, Toomey E, Blackwood $B$, et al. Personal protective equipment for preventing highly infectious diseases due to exposure to contaminated body fluids in healthcare staff. Cochrane Database Syst Rev. 2020 Apr;4:CD011621. PMID:32293717

14. Chughtai AA, Chen X, Macintyre CR. Risk of self-contamination during doffing of personal protective equipment. Am J Infect Control. 2018 Dec;46(12):1329-34. https://doi.org/10.1016/j. ajic.2018.06.003 PMID:30029796

15. Tabah A, Ramanan M, Laupland KB, Buetti N, Cortegiani A, Mellinghoff J, et al.; PPE-SAFE contributors. Personal protective equipment and intensive care unit healthcare worker safety in the COVID-19 era (PPE-SAFE): an international survey. J Crit Care. 2020 Oct;59:70-5. https://doi.org/10.1016/j.jcrc.2020.06.005 PMID:32570052

16. Navarro JR, Duque G, Ramírez E. Aprendizaje de habilidades sicomotoras. Bogotá: Sociedad Colombiana de Anestesiología y Reanimación S.C.A.R.E.; 2014.

17. Starkie T, Drake EJ. Assessment of procedural skills training and performance in anesthesia using cumulative sum analysis (cusum). Can J Anaesth. 2013 Dec;60(12):1228-39. https://doi. org/10.1007/s12630-013-0045-1 PMID:24242953

18. Hindman BJ, Dexter F, Kreiter CD, Wachtel RE. Determinants, associations, and psychometric properties of resident assessments of anesthesiologist operating room supervision. Anesth Analg. 2013 Jun;116(6):1342-51. https://doi.org/10.1213/ ANE.0b013e31828d7218 PMID:23558839

19. Fortea-Sanchis C, Escrig-Sos J. Técnicas de control de calidad en cirugía. Aplicación de las gráficas de control cumulative sum. Cir Esp (Engl Ed). 2019 Feb;97(2):65-70. https://doi.org/10.1016/j. cireng.2019.01.010 PMID:30686474

20. Dexter F, Ledolter J, Hindman BJ. Bernoulli Cumulative Sum (CUSUM) control charts for monitoring of anesthesiologists' performance in supervising anesthesia residents and nurse anesthetists. Anesth Analg. 2014 Sep;119(3):679-85. https://doi.org/10.1213/
ANE.0000000000000342 PMID:25137002

21. Konrad C, Schüpfer G, Wietlisbach M, Gerber H. Learning manual skills in anesthesiology: is there a recommended number of cases for anesthetic procedures? Anesth Analg. 1998 Mar;86(3):635-9. https://doi.org/10.1213/00000539199803000-00037 PMID:9495429

22. Norris A, McCahon R. Cumulative sum (CUSUM) assessment and medical education: a square peg in a round hole. Anaesthesia. 2011 Apr;66(4):250-4. https://doi.org/10.1111/j.13652044.2011.06692.x PMID:21401536

23. Miller GE. The assessment of clinical skills/competence/performance. Acad Med. 1990 Sep;65(9 Suppl):S63-7. https://doi. org/10.1097/00001888-199009000-00045 PMID:2400509

24. Asociación Médica Mundial. Declaración de Helsinki de la Asociación Médica mundial. Principios éticospara las investigaciones médicas en seres humanos. Fortaleza: 64.a Asamblea General de la AMM; 2013 (Citado 22 de agosto de 2020). Disponible de: https://www.wma.net/es/policies-post/declaracion-de-helsinkide-la-amm-principios-eticos-para-las-investigaciones-medicas-enseres-humanos/

25. Maestrea J, Rudolph J. Teorías y estilos de debriefing: el método con buen juicio como herramienta de evaluación formativa en salud. Rev Esp Cardiol. 2005;68(4):282-5. https://doi.org/10.1016/j. recesp.2014.05.018

26. Feinstein L, Sabates R, Tashweka MA, Sorhaindo A, Hammond $C$. What are the effects of education on health? In measuring the effects of education on health and civic engagement: proceedings of the copenhagen symposium - (c) oecd 2006. [citado: 21-08-2020]. Disponible en: https://www.oecd.org/education/ innovation-education/37425753.pdf

27. WHO. Rational use of personal protective equipment for coronavirus disease 2019 (COVID-19) Interim guidance 27 February 2020. [citado: 22-08-2020]. Disponible en: https://apps.who.int/ iris/bitstream/handle/10665/331215/WHO-2019-nCov-IPCPPE_ use-2020.1-eng.pdf 